\title{
Origin of the asymmetry of the wind driven halo observed in high-contrast images
}

\author{
F. Cantalloube ${ }^{1}$, E. H. Por ${ }^{2}$, K. Dohlen ${ }^{3}$, J.-F. Sauvage ${ }^{3,4}$, A. Vigan ${ }^{3}$, M. Kasper ${ }^{5}$, N. Bharmal ${ }^{6}$, \\ T. Henning ${ }^{1}$, W. Brandner ${ }^{1}$, J. Milli ${ }^{7}$, C. Correia ${ }^{3}$, and T. Fusco ${ }^{3,4}$
}

\author{
1 Max Planck Institute for Astronomy, Königstuhl 17, 69117 Heidelberg, Germany \\ e-mail: cantalloube@mpia.de \\ 2 Leiden Observatory, Leiden University, PO Box 9513, 2300 RA Leiden, The Netherlands \\ 3 Aix-Marseille Univ., CNRS, CNES, LAM, Marseille, France \\ 4 ONERA - The French Aerospace Lab, 92322 Châtillon, France \\ 5 European Southern Observatory (ESO), Karl-Schwarzschild-Str. 2, 85748 Garching, Germany \\ ${ }^{6}$ Centre for Advanced Instrumentation, Durham University, South Road, Durham DH1 3LE, UK \\ 7 European Southern Observatory (ESO), Alonso de Córdova 3107, Vitacura, Casilla, 19001 Santiago, Chile
}

Received 24 September 2018 / Accepted 27 October 2018

\begin{abstract}
The latest generation of high-contrast instruments dedicated to exoplanets and circumstellar disk imaging are equipped with extreme adaptive optics and coronagraphs to reach contrasts of up to $10^{-4}$ at a few tenths of arcseconds in the near-infrared. The resulting image shows faint features, only revealed with this combination, such as the wind driven halo. The wind driven halo is due to the lag between the adaptive optics correction and the turbulence speed over the telescope pupil. However, we observe an asymmetry of this wind driven halo that was not expected when the instrument was designed. In this letter, we describe and demonstrate the physical origin of this asymmetry and support our explanation by simulating the asymmetry with an end-to-end approach. From this work, we find that the observed asymmetry is explained by the interference between the AO-lag error and scintillation effects, mainly originating from the fast jet stream layer located at about $12 \mathrm{~km}$ in altitude. Now identified and interpreted, this effect can be taken into account for further design of high-contrast imaging simulators, next generation or upgrade of high-contrast instruments, predictive control algorithms for adaptive optics, or image post-processing techniques.
\end{abstract}

Key words. instrumentation: adaptive optics - instrumentation: high angular resolution - atmospheric effects techniques: image processing - methods: data analysis - infrared: planetary systems

\section{Introduction}

With the arrival of the new generation of high-contrast imaging (HCI) instruments equipped with extreme adaptive optics (XAO) and advanced coronagraphs, dedicated to exoplanet and circumstellar disk imaging, we can now visualize optical effects that were expected but never before revealed. On $8 \mathrm{~m}$ class telescopes, instruments such as VLT/SPHERE (Beuzit et al. 2008), Gemini/ GPI (Macintosh et al. 2008), Clay/MagAO-X (Close et al. 2012; Males et al. 2014), and Subaru/SCExAO (Jovanovic et al. 2015) are equipped with XAO, providing a Strehl ratio of up to $95 \%$ in the near-infrared, and coronagraphs, providing a raw contrast of up to $10^{-4}$ at a few hundred milliarcseconds (mas). Images obtained with these instruments show features such as the correction radius of the $\mathrm{XAO}$, the deformable mirror actuator grid print-through, the bright central spot due to diffraction effects in the Lyot coronagraph (Poisson spot or Arago spot), and the wind driven halo due the temporal lag between the application of the $\mathrm{XAO}$ correction and the evolving turbulence. All these features were expected and taken into account when designing and simulating the instrument.

However, some unexpected features are also visible within HCI images: the wind driven halo often shows an asymmetry, one wing being brighter and broader than the other, and the point-spread function (PSF) sometimes breaks up, leading to catastrophic loss of performance. While the latter, known as the low wind effect, is described elsewhere (Milli et al. 2018), describing and understanding the asymmetric wind driven halo, which also limits the high-contrast capabilities of the instrument, is the object of this letter.

We first describe qualitatively the observed asymmetry of the wind driven halo (Sect. 2). Based on these observations, we propose an explanation and derive its mathematical demonstration (Sect. 3). To prove our interpretation, we perform end-to-end simulations taking into account the optical effect that generates the asymmetry and checked that the asymmetry indeed varies as expected with the parameters upon which it depends (Sect. 4).

\section{Description of the observed asymmetry}

The wind driven halo (WDH) is the focal plane expression of the AO servolag error (also often referred to as temporal bandwidth error). The AO-lag temporal error appears when the turbulence equivalent velocity above the telescope pupil (defined via the coherence time $\tau_{0}$, up to a few tens of milliseconds under good conditions) is faster than the adaptive optics correction loop frequency (being about $1.4 \mathrm{kHz}$ for SAXO, the XAO of SPHERE, 


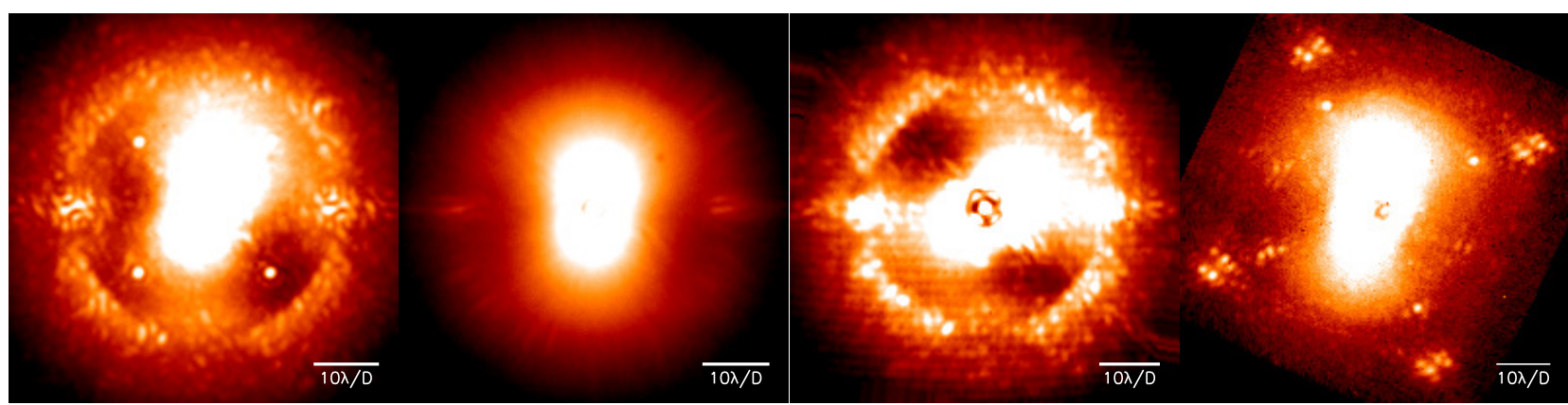

Fig. 1. Coronagraphic focal plane images showing the asymmetry of the wind driven halo. Left panel: one exposure obtained with SPHEREIRDIS (H2 band, $1.593 \mu \mathrm{m}, \Delta \lambda \approx 53 \mathrm{~nm}$ ). Middle left panel: one exposure obtained with SPHERE-IFS (second channel of YH mode, $0.991 \mu \mathrm{m}$, $\Delta \lambda \approx 30 \mathrm{~nm}$ ). Middle right panel: one exposure obtained with SPHERE-IRDIS in broadband ( $H$ band, $1.625 \mu \mathrm{m}, \Delta \lambda \approx 291 \mathrm{~nm}$ ). Right panel: one exposure obtained with GPI (second channel of YH mode, $1.503 \mu \mathrm{m}, \Delta \lambda \approx 45 \mathrm{~nm}$ ). The images are purposely stretched in intensity to highlight the asymmetry (log scale).

Petit et al. 2014). Using a coronagraph and a sufficiently long detector integration time (DIT) reveals, in the focal plane, the starlight diffracted by this specific error. As a consequence, the PSF is elongated along the projected wind direction, making a butterfly-shaped halo appear on the images. By definition, this aberration being a phase shift in the pupil plane, it must be symmetric in the focal plane. In practice, however, we observe an asymmetry of the WDH along its axis: one wing being smaller and fainter than the other.

The images obtained with SPHERE and $\mathrm{GPI}^{1}$ (see Fig. 1) show the asymmetry of the WDH. To highlight the asymmetry, Fig. 2 shows the radial profile along the wind direction and the azimuthal profile at $6 \lambda / \mathrm{D}$ of the SPHERE-IRDIS image presented in Fig. 1 (left).

By definition, the WDH is produced by high wind speed turbulent layers. It has been confirmed that it is mainly triggered by the high-altitude jet stream layer, located in a narrow region of the upper troposphere, at about $12 \mathrm{~km}$ above sea level $(200 \mathrm{mbar})$ and with a wind speed from $20 \mathrm{~m} \mathrm{~s}^{-1}$ to $50 \mathrm{~m} \mathrm{~s}^{-1}$ (Tokovinin et al. 2003; Osborn \& Sarazin 2018). Madurowicz et al. (2018) demonstrated it by correlating the WDH direction with the wind direction at different altitude given by turbulence profiling data for the whole GPIES survey data (Macintosh 2013). A forthcoming paper, which draws the same conclusion, will similarly analyse the WDH within SPHERE data.

Focal plane asymmetries can only be created by combining phase and amplitude aberrations. As we observed that the asymmetry is pinned to the servolag signature (butterfly shape), we considered that it may be caused by the interaction between servolag errors and amplitude errors created by scintillation, where the phase errors generated by high atmospheric layers propagate into amplitude errors following Fresnel's propagation laws.

\section{Interference between scintillation and temporal error}

In the following we provide an analytical demonstration that the combination of two well-known effects, the AO loop delay (servolag error) and scintillation (amplitude error), which indeed create the asymmetric starlight distribution observed in the highcontrast images.

\footnotetext{
1 SPHERE images have been published in respectively Bonnefoy et al. (2018), Wahhaj et al. (2015), Samland et al. (2017), and GPI data in Rameau et al. (2016).
}
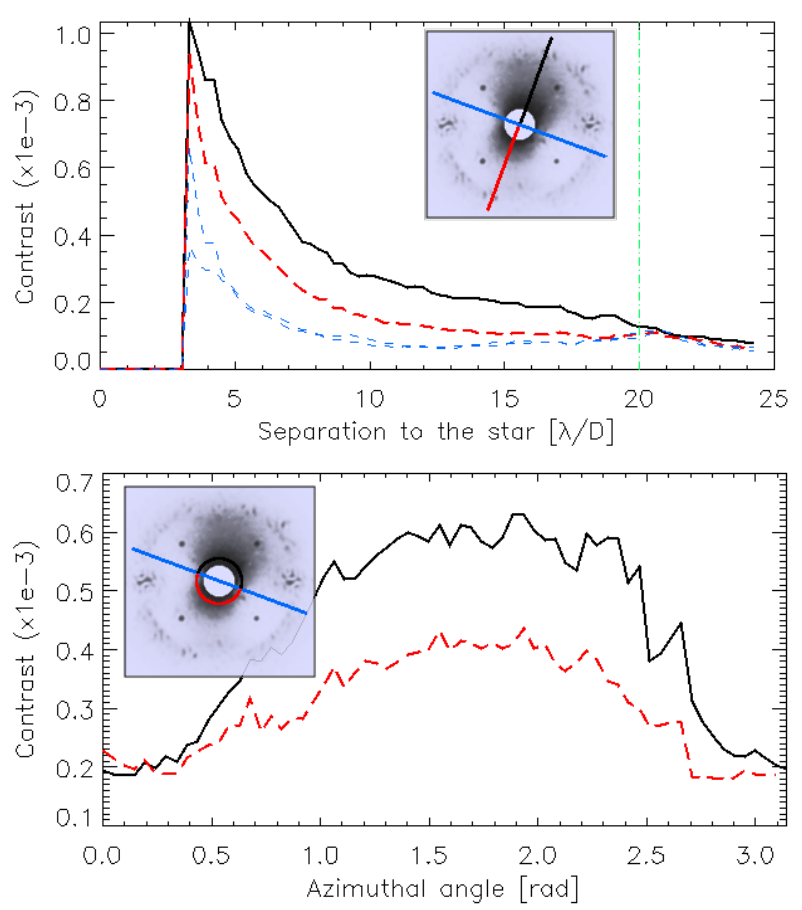

Fig. 2. Profiles of the wind driven halo showing the asymmetry in a SPHERE-IRDIS image. The solid line is along the brighter and bigger wing; the dashed line is along the fainter and smaller wing. Top panel: radial profile along the WDH direction (black solid and red long-dashed lines) and its perpendicular direction (blue dashed lines). The DM cutoff frequency is at $20 \lambda / \mathrm{D}$ (green dot-dashed line). Bottom panel: azimuthal profile at $6 \lambda / \mathrm{D}$ from the star.

In the pupil plane, the electric field can be written as

$E=(1-\epsilon) \cdot \mathrm{e}^{i \phi}$,

where $\epsilon$ is the amplitude aberration and $\phi$ the phase aberration. An adaptive optic system measures the phase $\phi(t)$ at a given time $t$ via the wavefront sensor (WFS) and corrects it using a deformable mirror (DM). However, between the analysis of the WFS information taken at an instant $t$ and the command sent to the DM at an instant $t+\Delta t$, if the incoming turbulent phase has varied during $\Delta t$, a temporal phase error will remain (the AO servolag error). As a general rule, this absolute time delay $\Delta t$ varies with both the AO-loop gain and the AO-loop speed and is intrinsic to any $\mathrm{AO}$ system. The remaining phase error $\Delta \phi$ can 
be written as a function of this absolute time delay $\Delta t$ following (in a closed loop system)

$\Delta \phi=\phi(t)-\phi(t-\Delta t) \sim \Delta t \phi^{\prime}$,

where $\phi^{\prime}$ is the time derivative of the phase. This approximation is valid for spatial frequencies affected by the servolag error, that is to say much lower than $1 /\left(v_{\text {wind }} \cdot \Delta t\right)$ under the frozen flow hypothesis (i.e. only the wind speed is responsible for the turbulent phase variation).

Thus, after the AO correction, the electric field becomes

$\Delta E=(1-\epsilon) \cdot \mathrm{e}^{i\left(\Delta t \phi^{\prime}\right)}$,

which, under the approximation of the small phase and small amplitude errors, simplifies to

$\Delta E \simeq(1-\epsilon) \cdot\left(1+i\left(\Delta t \cdot \phi^{\prime}\right)\right) \sim 1-\epsilon+i\left(\Delta t \phi^{\prime}\right)$.

Seen through a perfect coronagraph (the patterns exclusively due to diffraction effects of a plane wavefront by the entrance pupil are entirely removed), the post-AO electric field $\Delta E_{c}$ is transformed into

$\Delta E_{c} \sim-\epsilon+i \Delta t \phi^{\prime}$.

The Earth's turbulent atmosphere is present to different degrees throughout the three dimensions of the atmosphere. Fresnel propagation translates phase variations in the upper atmosphere into amplitude variations via the Talbot effect, creating the so-called scintillation. By the formalism of Zhou \& Burge (2010), the phase variations in an atmospheric layer located at altitude $z$ produces an amplitude distribution at the telescope pupil of

$\epsilon=\sin \left(2 \pi \frac{z}{z_{T}}\right) \phi$

where $z_{T}$ is the Talbot length, defined as $z_{T} \doteq 2 /\left(f^{2} \lambda\right)$, where $f$ is the spatial frequency and $\lambda$ the wavelength. The distance at which a pure phase error is fully converted into a pure amplitude error is at one quarter of the Talbot length ${ }^{2}$. For SPHERE, the highest imaging wavelength is $2.2 \mu \mathrm{m}$ ( $K$ band) and the highest corrected spatial frequency is $2.5 \mathrm{~m}^{-1}$, given by the DM inter-actuator spacing $(40 \times 40$ actuators over the $8 \mathrm{~m}$ diameter telescope pupil), yielding a minimum distance of about $36 \mathrm{~km}$ altitude, which is above the highest turbulence layers. This explains why, for both GPI and SPHERE, this effect was neglected when designing the instrument.

Adding the scintillation into the coronagraphic post-AO electric field of Eq. (5) gives

$\Delta E_{c} \sim-\sin \left(2 \pi \frac{z}{z_{T}}\right) \phi+i \Delta t \phi^{\prime}$.

The resulting intensity observed at the focal plane $\left(I_{c}\right)$ is, within the Fraunhofer framework, the squared modulus of the Fourier transform of the electric field $\Delta E_{c}$ :

$I_{c}=\left|\mathrm{FT}\left[\Delta E_{c}\right]\right|^{2}=\left|-\sin \left(2 \pi \frac{z}{z_{T}}\right) \mathrm{FT}[\phi]+i \Delta t \operatorname{FT}\left[\phi^{\prime}\right]\right|^{2}$

with $\mathrm{FT}\left[\phi^{\prime}\right]=\frac{\partial \mathrm{FT}[\phi]}{\partial t}=\mathrm{FT}^{\prime}[\phi]$ being the time derivative of the Fourier transform of the phase. If we assume an arbitrary phase whose general expression can be written $\phi=\exp (i 2 \pi f \cdot r), f$ being the spatial frequency and $r$ the position, then by making the change of variable $r \leftarrow r+\Delta r$ where we define the beam shift

\footnotetext{
2 Under the hypothesis of a monochromatic propagation within infinite pupil extent and small phase approximation.
}

factor $\Delta r=\left(v_{\text {wind }} . \Delta t\right)$ to account for the servolag shift (under the frozen flow hypothesis), Eq. (8) becomes

$$
\begin{aligned}
I_{c} & =\left|-\sin \left(2 \pi \frac{z}{z_{T}}\right) \operatorname{FT}[\phi]-2 \pi f v_{\text {wind }} \Delta t \mathrm{FT}[\phi]\right|^{2} \\
& =|\mathrm{FT}[\phi]|^{2}\left(\sin \left(2 \pi \frac{z}{z_{T}}\right)+2 \pi f \Delta r\right)^{2},
\end{aligned}
$$

where $|\mathrm{FT}[\phi]|^{2}$ is by definition the power spectral density of the turbulent phase and $\Delta r$ represents the physical spatial shift between the turbulent layer and the AO correction. Developing Eq. (9) leads to an asymmetric function of the spatial frequency $f: I_{c}$ indeed shows an asymmetric distribution of light in the high-contrast images with respect to the centre, originating from interference. Therefore, the intensity of each wing of the $\mathrm{WDH}$ can be written, respectively for constructive and destructive interference $I_{+}$and $I_{-}$, as follows:

$I_{+}=|\mathrm{FT}[\phi]|^{2}\left(\sin \left(2 \pi \frac{z}{z_{T}}\right)+2 \pi f \Delta r\right)^{2}$
$I_{-}=|\mathrm{FT}[\phi]|^{2}\left(\sin \left(2 \pi \frac{z}{z_{T}}\right)-2 \pi f \Delta r\right)^{2}$.

We thus demonstrate that a temporal phase shift (from temporal delay of the AO loop) between phase error (from the atmospheric turbulence) and amplitude error (from the scintillation effect) creates an asymmetry pinned to the wind driven halo in the focal plane image.

We can define the relative asymmetry factor, $F_{\text {asymmetry, }}$ as the normalized difference between these two intensities:

$$
\begin{aligned}
F_{\text {asymmetry }} & \doteq \frac{I_{+}-I_{-}}{I_{+}+I_{-}} \\
& =\frac{2 \sin \left(2 \pi \frac{z}{z_{T}}\right) f \Delta r}{\left(\sin \left(2 \pi \frac{z}{z_{T}}\right)\right)^{2}+(f \Delta r)^{2}} .
\end{aligned}
$$

This factor is thus between 0 (no asymmetry) and 1 (all the light is spread in only one wing $)^{3}$.

With current HCI instruments, the WDH has a typical contrast of $10^{-4}$ (see Fig. 2), whereas the scintillation has a typical contrast of $10^{-6}$ (Tatarski 2016) so we can ignore the scintillation term in the denominator and simplify to $\sin \left(\frac{z}{z_{T}}\right) \sim \frac{z}{z_{T}}$, which yields, after replacing the Talbot length by its expression, the following approximation:

$F_{\text {asymmetry }}=\frac{z f \lambda}{v_{\text {wind }} \Delta t}+O\left(\left(\frac{z f \lambda}{v_{\text {wind }} \Delta t}\right)^{2}\right)$.

We consequently expect the asymmetry factor to grow linearly with the spatial frequency, and therefore with the angular separation to the star. From this demonstration we can already infer a few effects. First, as the interference is taking place between the turbulence residuals and the AO correction lag, any type of coronagraph will reveal the asymmetry of the WDH. Second, even though the Talbot length is $36 \mathrm{~km}$ while the jet stream layer is at an altitude of $12 \mathrm{~km}$, the propagation distance is sufficient to

\footnotetext{
3 The asymmetry factor is maximum $\left(F_{\text {asymmetry }}=1\right)$ when the numerator is equal to $1 / 2$ (i.e. $\sin \left(2 \pi \frac{z}{z_{T}}\right) \cdot f \Delta r=1$ : the amplitude error is fully correlated with the phase error), and is minimum $\left(F_{\text {asymmetry }}=0\right)$ when the numerator is null (null wind speed, no temporal lag: there is no wind driven halo) or equal to infinity (there is no correlation at all between amplitude error and phase error).
} 
convert a small fraction of the phase error into amplitude error and therefore produce the observed asymmetry. Consequently, the higher the altitude of the fast layer, the more asymmetry is produced. On the contrary, the ground layer does not produce this asymmetry. Third, knowing that the amplitude errors are only due to the turbulence, whereas the delayed phase error is due to both the wind speed and the AO loop correction speed, the asymmetry varies with temporal parameters as follows: (i) if the AO loop delay $\Delta t$ increases (e.g. the AO loop is slower) we lose the correlation between the amplitude errors and the delayed phase errors, making the asymmetry smaller; (ii) if the wind speed $v_{\text {wind }}$ is higher, the correlation between the amplitude error and the delayed phase error decreases, making the asymmetry smaller. In other word, if the beam shift $\Delta r$ between the turbulent layer and the AO correction increases, the correlation decreases and so does the asymmetry. Finally, we expect this asymmetry to increase with wavelength as the Talbot length is inversely proportional to wavelength, while the other parameters are independent of wavelength.

As a consequence, an observation site such as Mauna Kea, which suffers less from jet stream compared to observatories located at Paranal in Chile (e.g. Sarazin et al. 2003), would be beneficial to avoid the wind driven halo ${ }^{4}$ in the high-contrast images, and the subsequent asymmetry which may arise depending on the AO correction setting and the speed of the highaltitude turbulent layers.

\section{Simulations of the effect}

In the following, we describe a numerical simulation of an idealized AO system reacting to a simplified atmosphere with a single, high-altitude turbulence layer. The goal is to explore the connection between servolag, scintillation, and the occurrence (or absence) of an asymmetric WDH. The simulations are conducted using the HCIPy package (Por et al. 2018), which is available as open-source software on GitHub ${ }^{5}$.

We simulated a single atmospheric layer at the altitude of the jet stream, which is then moved across the telescope aperture according to the frozen-flow hypothesis. The light is propagated from the layer to the ground using an angular-spectrum Fresnel propagation code. This light is sensed using a noiseless WFS, which in turn is used to drive a DM. An integral controller with a gain of 0.5 is assumed. The flattened wavefront is then propagated through a perfect coronagraph (Cavarroc et al. 2006) before being focused onto the science camera. We carry out 500 independent short-exposure simulations, which are then stacked to form the final long-exposure image. A list of the nominal simulation parameters can be found in Table 1 .

Figure 3 shows the coronagraphic simulated images obtained with or without AO lag and with or without scintillation. As expected, only the combination of both amplitude error and AO servolag error leads to an asymmetric WDH.

Figure 4 shows the radial profile of the simulated images along the wind direction (top) and the corresponding asymmetry factor as defined at Eq. (12) (bottom), as a function of the separation to the star, where we observe that the asymmetry grows linearly with the separation. We also demonstrate that the scintil-

\footnotetext{
4 The Subaru/SCExAO high-contrast images do not show the wind driven halo and its asymmetry. This might also be explained by the use of predictive control algorithm based on machine-learning techniques, which aims to eliminate the servolag error (Males \& Guyon 2018; Guyon \& Males 2017).

5 https://github.com/ehpor/hcipy
}

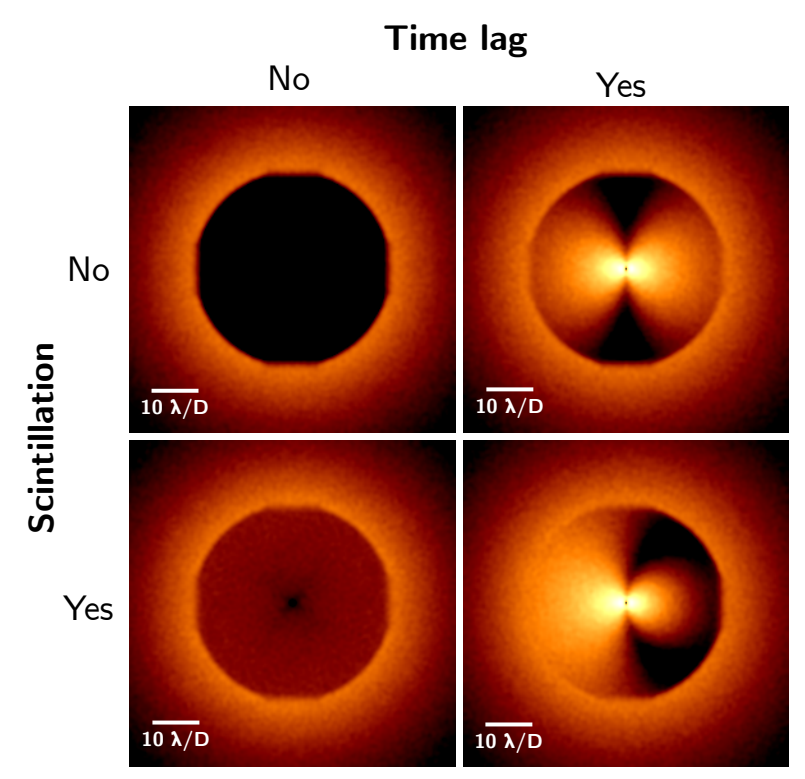

Fig. 3. Simulated images using HCIPy for the parameters gathered in Table 1 . The images with no time lag were produced with an infinite AO loop speed. Only a time-lagged WDH and scintillation yields an asymmetric coronagraphic PSF. The images have been stretched in intensity to highlight the asymmetry and scintillation (log scale).

Table 1. Nominal set of parameters used for our simulations.

\begin{tabular}{ll}
\hline \hline Parameter name & Value \\
\hline Wavelength & $2.2 \mu \mathrm{m}(K$ band $)$ \\
Pupil diameter & $8 \mathrm{~m}$ \\
Seeing & $r_{0}=20 \mathrm{~cm}$ at $500 \mathrm{~nm}$ \\
Outer scale & $22 \mathrm{~m}$ \\
Jet stream height & $12 \mathrm{~km}$ \\
Jet stream velocity & $30 \mathrm{~m} \mathrm{~s}^{-1}$ \\
AO system loop speed & $1380 \mathrm{~Hz}$ \\
AO system controller & Integral control \\
AO system loop gain & 0.5 for all modes \\
Corrected modes & 1000 modes \\
Number of actuators & $40 \times 40$ rectangular grid \\
Influence functions & Gaussian with $\sigma=22 \mathrm{~cm}$ projected \\
Coronagraph & Perfect (Cavarroc et al. 2006) \\
Wavefront sensor & Noiseless \\
\hline
\end{tabular}

lation from the jet stream layer at $12 \mathrm{~km}$ altitude is enough to create the asymmetry of the wind driven halo and that lower altitude layers create less asymmetry. As expected from the approximation of Eq. (14), our simulations also show that the asymmetry is stronger when the wind speed decreases or when the AO loop frequency decreases (for a fixed AO loop gain). We also checked that the asymmetry factor is indeed higher at longer wavelengths.

In a forthcoming paper we will compare this analysis to onsky images obtained with SPHERE, which involves isolating the contribution of the WDH in the image since other error terms are hiding these trends.

\section{Conclusions}

In this letter we pointed out the presence of an asymmetry of the wind driven halo that is revealed in high-contrast images. We described and demonstrated its origin as being due to 

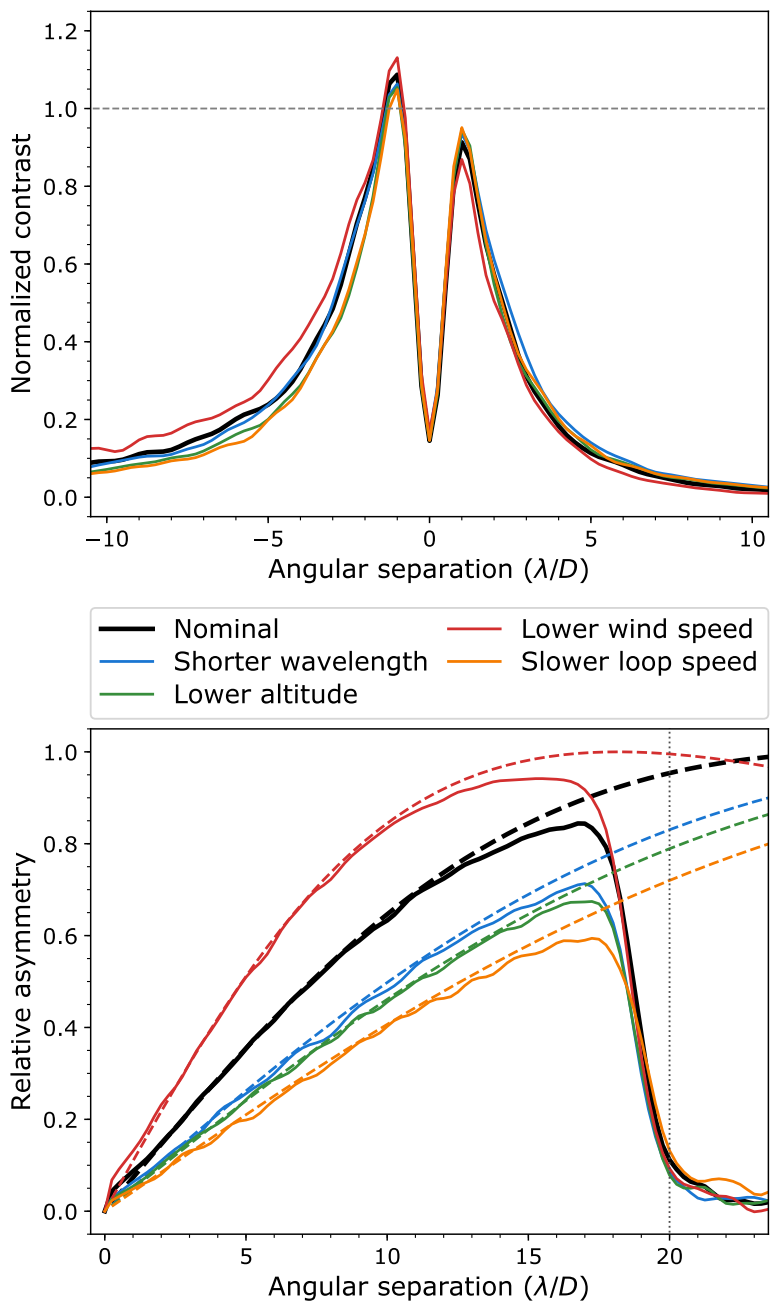

Fig. 4. Radial profiles along the wind direction (top panel) and asymmetry factor as defined in Eq. (12) (bottom panel) for the simulated data sets. The solid black line uses the nominal parameters from Table 1 (image shown in the bottom right of Fig. 3). Other lines differ in one parameter playing a role in the asymmetry: shorter wavelength $(H$-band, $\lambda=1.6 \mu \mathrm{m}$, blue line), lower altitude $(z=8 \mathrm{~km}$, green line), lower wind speed $\left(v_{\text {wind }}=20 \mathrm{~m} \mathrm{~s}^{-1}\right.$, red line), and slower AO loop frequency

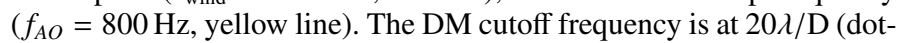
ted grey line). In the bottom plot, solid lines indicate the asymmetry from the simulated images and dashed lines show the prediction from Eq. (14).

interference between AO correction lag (delayed phase error) and scintillation (amplitude errors). We supported our demonstration by simulating this effect using an end-to-end simulator. From these simulations we confirmed the expected behaviour of the asymmetry with different atmospheric turbulence conditions, $\mathrm{XAO}$ correction, and imaging wavelength. We further demonstrated that the jet stream layer is the main culprit for this aberration since it is responsible for both servolag error (being a fast layer) and scintillation (being a high-altitude layer). Therefore, an observing site with weak or no jet stream would get around this aberration.

While the current letter focuses on exploring the origin of the wind driven halo asymmetry so as to better understand our current observations and AO systems for future designs, a more quantitative analysis of its implication on high-contrast imaging capabilities and potential mitigation strategies will be detailed in a separate paper. Indeed, the servolag error, when present, is now one of the major effects limiting the high-contrast capabilities of the current instruments (along with the low wind effect, the noncommon path aberrations, and residual tip-tilt errors). Knowing that this wind driven halo shows an asymmetry makes it more difficult to deal with in post-processing (as using for instance the residual phase structure functions yields a symmetric phase error or that most filters have a symmetric effect).

Now that this effect has been acknowledged and demonstrated, the next step is to take it into account within end-to-end XAO simulators (e.g. COMPASS or SOAPY, Gratadour et al. 2014; Reeves 2016) or analytical simulators (e.g. PAOLA, Jolissaint 2010) and more generally in XAO error budgets, when used in the HCI framework. This study gives insights into the instrument operations, essential to designing optimal post-processing techniques or AO predictive control tools, which both aim to eliminate the servolag error signature (e.g. Males \& Guyon 2018; Correia 2018. This effect is also important in order to design the next generation of high-contrast instruments (e.g. MagAOX, Close et al. 2018 or giant segmented mirror telescopes instruments dedicated to $\mathrm{HCI}$ ) or to lead the upgrades of existing high-contrast instruments (e.g. GPI or SPHERE, Chilcote et al. 2018; Beuzit et al. 2018), for instance by adding a second DM to correct for the scintillation.

Acknowledgements. We would like to thank the anonymous referee for the comments which helped clarify the letter. The authors would also like to thank J. Lozi and O. Guyon for the discussions about the wind driven halo in the Subaru/SCExAO images. EHP acknowledges funding by The Netherlands Organisation for Scientific Research (NWO) and the São Paulo Research Foundation (FAPESP). NAB acknowledges UK Research \& Innovation Science and Technologies Facilities Council funding (ST/P000541/1).

\section{References}

Beuzit, J.-L., Feldt, M., Dohlen, K., et al. 2008, Proc. SPIE, 7014, 701418 Beuzit, J., Mouillet, D., Fusco, T., et al. 2018, Proc. SPIE, 10703, 107031P Bonnefoy, M., Perraut, K., Lagrange, A.-M., et al. 2018, A\&A, 618, A63

Cavarroc, C., Boccaletti, A., Baudoz, P., Fusco, T., \& Rouan, D. 2006, A\&A, 447,397

Chilcote, J., Bailey, V., De Rosa, R., et al. 2018, Proc. SPIE, 10702, 1070244

Close, L. M., Males, J. R., Kopon, D. A., et al. 2012, Adapt. Opt. Syst. III, 8447, $84470 X$

Close, L. M., Males, J. R., Durney, O., et al. 2018, Proc. SPIE, 10703, 10703K Correia, C. M. 2018, Proc. SPIE, 7736, 77361J

Gratadour, D., Puech, M., Vérinaud, C., et al. 2014, in Adaptive Optics Systems IV, Int. Soc. Opt. Photonics, 9148, 91486 O

Guyon, O., \& Males, J. 2017, AJ, accepted, [arXiv:1707.00570]

Jolissaint, L. 2010, J. Eur. Opt. Soc., 5, 10055

Jovanovic, N., Martinache, F., Guyon, O., et al. 2015, PASP, 127, 890

Macintosh, B. 2013, The Gemini Planet Imager Exoplanet Survey, NASA OSS Proposal

Macintosh, B. A., Graham, J. R., Palmer, D. W., et al. 2008, Proc. SPIE, 7015, 701518

Madurowicz, A., Macintosh, B., Ruffio, J.-B., et al. 2018, Proc. SPIE, 10703, $107036 \mathrm{E}$

Males, J. R., \& Guyon, O. 2018, Instrum. Syst., 4, 019001

Males, J. R., Close, L. M., Morzinski, K. M., et al. 2014, ApJ, 786, 32

Milli, J., Kasper, M., Bourget, P., et al. 2018, Proc. SPIE, 10703, 107032A

Osborn, J., \& Sarazin, M. 2018, MNRAS, 480, 1278

Petit, C., Sauvage, J.-F., Fusco, T., et al. 2014, Proc. SPIE, 9148, 91480 O

Por, E. H., Haffert, S. Y., Radhakrishnan, V. M., et al. 2018, Proc. SPIE, 10703, 1070342

Rameau, J., Nielsen, E. L., De Rosa, R. J., et al. 2016, ApJ, 822, L29

Reeves, A. 2016, Int. Soc. Opt. Photonics, 9909, 99097F

Samland, M., Mollière, P., Bonnefoy, M., et al. 2017, A\&A, 603, A57

Sarazin, M. S., Graham, E., Beniston, M., \& Riemer, M. 2003, Int. Soc. Opt. Photonics, 4840, 291

Tatarski, V. I. 2016, Wave Propagation in a Turbulent Medium (Courier Dover Publications)

Tokovinin, A., Baumont, S., \& Vasquez, J. 2003, MNRAS, 340, 52

Wahhaj, Z., Cieza, L. A., Mawet, D., et al. 2015, A\&A, 581, A24

Zhou, P., \& Burge, J. H. 2010, Appl. Opt., 49, 535 